\title{
Balò's concentric sclerosis: still to be considered as a variant of multiple sclerosis?
}

\author{
Anna M. Pietroboni ${ }^{1} \cdot$ Andrea Arighi $^{1} \cdot$ Milena A. De Riz ${ }^{1}$ Laura Ghezzi ${ }^{1}$. \\ Alberto Calvi $^{1} \cdot$ Sabrina Avignone $^{2} \cdot$ Elisa Scola $^{2} \cdot$ Daniela Galimberti $^{1}$. \\ Fabio Triulzi ${ }^{2} \cdot$ Elio Scarpini ${ }^{1}$
}

Received: 26 March 2015/Accepted: 16 June 2015/Published online: 25 June 2015

(C) Springer-Verlag Italia 2015

\begin{abstract}
Balò's concentric sclerosis (BCS) is considered a rare demyelinating disease and regarded as an aggressive variant of multiple sclerosis (MS). We describe three cases (one male and two females) with neuroimaging features suggestive of BCS and heterogeneous symptoms, with benign long-term clinical course upon treatment with natalizumab and fingolimod. Neurological examination, blood and cerebrospinal fluid analyses, brain and spinal cord magnetic resonance imaging (MRI) and brain proton magnetic resonance spectroscopy were performed. At onset, patient \#1 showed predominant cognitive impairment with consciousness disturbances; patient \#2 presented with left hemiparesis; patient \#3 demonstrated hesitance in speech and in written word production, along with right central facial palsy. All patients showed the typical MRI changes associated with BCS, such as concentric rings or a whorled appearance on T2-weighted and contrast-enhanced T1-weighted images. They were treated with high dosage i.v. steroid with clinical improvement and followed-up for 3 years with different clinical course. Two patients fulfilled the revised McDonald criteria for MS and received preventive therapy, natalizumab and fingolimod, respectively, whereas the third patient is still stable without clinical and radiological evolution. All of them did not have new exacerbations or MRI lesions over 2-4 year follow-up. Our descriptions demonstrate the heterogeneity of clinical
\end{abstract}

Anna M. Pietroboni

pb.anna@libero.it

1 Neurology Unit, Department of Pathophysiology and Transplantation, "Dino Ferrari" Center, Fondazione Cà Granda, IRCCS Ospedale Maggiore Policlinico, University of Milan, Milan, Italy

2 Division of Neuroradiology, Fondazione Cà Granda, IRCCS Ospedale Maggiore Policlinico, Milan, Italy presentation of BCS. Moreover, these case reports suggest that BCS may neither be rapidly progressive nor fatal and may be considered part of the MS spectrum. In line with this hypothesis, current treatments for MS were effective in our patients.

Keywords Balò's concentric sclerosis (BCS) · Multiple sclerosis (MS) · Clinically isolated syndrome (CIS)

\section{Introduction}

Balò's concentric sclerosis (BCS) is a rare demyelinating disease and it is regarded as one of the atypical forms of multiple sclerosis (MS) [1], presenting an aggressive, and often fatal, clinical course [2]. The most commonly reported clinical signs of BCS include cognitive or behavioural dysfunction, headache and/or seizures [3] and the main differential diagnoses are tumefactive demyelination, acute disseminated encephalomyelitis, central nervous system tumours, cerebral abscess and lymphoma. BCS was once a post-mortem diagnosis. However, the advent of MRI imaging [4] has led to better understanding of the disease, and it is now recognized that many patients can nearly completely recover after an attack [5].

Hereby, we report three cases presenting with typical neuroimaging features for BCS, fast clinical progression at the onset, but significantly different and non-fatal clinical evolutions.

\section{Case reports}

Patient \#1 A 27-year-old woman presented with insidious onset of ideomotor impairment and progressively increasing consciousness disturbance. Neurological examination 
showed psychomotor slowdown, right hemineglect and ideomotor apraxia. Cerebrospinal fluid (CSF) analyses were normal, and oligoclonal bands (OB) were absent. Brain magnetic resonance imaging (MRI) showed two T2 hyperintense lesions with open ring enhancement in the left hemisphere, the larger one presenting lamellar concentric features (Fig. 1a). Spinal cord MRI was normal. Spectroscopy showed decrease of $N$-acetylaspartate and increase of choline, as described in other cases of BCS [1, 6].

She was treated with high dosage i.v. steroid with fast clinical improvement. A second brain MRI showed a marked reduction of the size of the lesions, the resolution of the mass effect and of the ring of enhancement described above (Fig. 1a). Brain MRI at 3 months proved a further reduction of left hemispheric lesions.

Ten months after discharge, she developed an acute attack, consisting of postural instability. At MRI, MS-like lesions appeared (Fig. 1b). Two lesions were gadolinium enhancing (Fig. 1c). Since she fulfilled the criteria for MS and in consideration of the rather severe onset of the disease, immunosuppressive therapy with natalizumab was started, as recently described in a few other BCS cases [5]. Neither new relapses nor new lesions appeared at MRI after 4-year follow-up.

Patient \#2 A 39-year-old woman presented with insidious onset of left hemiparesis. Neurological examination showed left hemiplegia and homolateral proprioceptive disturbances. Blood and CSF analyses were normal, and OB were absent. Brain MRI revealed a T2 hyperintense non-enhancing lesion, with lamellar concentric features (Fig. 1d). Spectroscopy showed decrease of $\mathrm{N}$-acetylaspartate peak, mild increase of choline peak and the presence of lactate peak (Fig. 1e). Spinal cord MRI was normal. She was treated with high dosage i.v. steroid with clinical improvement. A second brain MRI did not show new lesions. At 20-month clinical follow-up, the patient was stable and her diagnosis remained that of clinically isolated syndrome (CIS).

Patient \#3A 31-year-old man presented with aphasia that developed within the previous 10 days. He reported an episode of lower limb paraesthesia, which occurred 2 months before hospitalization and regressed spontaneously after several days. Neurological examination proved hesitance in speech and in written word production, along with right central facial palsy. CSF analyses revealed the presence of OB. Brain MRI showed two large lesions with lamellar concentric features (Fig. 1f). Both were gadolinium ring-enhancing lesions. Spinal cord MRI showed cervical and dorsal non-enhancing MS plaques as well (Fig. 1f). Taken together, these data were enough to fulfil MS criteria. Spectroscopy findings were consistent with those described in other cases of BCS $[1,6]$.
Symptoms improved after a 5-day course of high dosage i.v. steroid. Follow-up showed a new non-enhancing MRI lesion and a clinical relapse (sensitive), which spontaneously regressed in few days. The presence of several spinal lesions at MRI, suggesting a quite aggressive course of the disease, prompted us to treat the patient with fingolimod, which was started soon after with no further relapses over 1-year follow-up.

\section{Discussion}

BCS has generally been regarded as a different entity from MS, in reason of poor prognosis, neuropathological lesions (alternating lamellae of demyelination and normal myelination) and absence of $\mathrm{OB}$ at CSF analysis [3]. This view has recently been questioned, although the boundaries between BCS and MS remain blurred [5, 6].

Typically, the cerebral BCS lesions detected by MRI scan are large with onion bulb-like pattern. Indeed, MS too can present with tumour-like lesions at brain MRI (1-2/ 1000 cases), presenting with a large intracranial lesion, perilesional oedema and ring enhancement with gadolinium contrast [7], but without the histological and radiological features described as typical for BCS. Recently, it has been reported in two close cases with BCS and tumefactive demyelination occurring in the same patient [8].

In support of the hypothesis that BCS is a manifestation of the MS spectrum, half of the patients who present with a Balò-like lesion have typical MS lesions elsewhere on their MRI scan. Furthermore, Balò lesions can occur during the clinical course of otherwise conventional relapsing-remitting MS on the one hand and small concentric bands of demyelinated and myelinated tissue are also found in acute multiple sclerosis lesions on the other [9]. At last, some patients with Balò lesions develop MS over time [5].

Our cases demonstrate the heterogeneity of clinical presentation of BCS, as part of MS clinical spectrum. In accordance with the recent observation that an increasing number of BCS cases show prolonged survival or spontaneous remission [10] our patients showed a relatively benign course during medium-term follow-up.

These case reports suggest that BCS may be heterogeneous in the clinical and radiological presentation and can show up either as a MS onset or throughout an already overt MS.

Finally, it may be argued that the typical MRI findings (traditionally linked with BCS as highly specific) could be regarded as being nothing but one of the several patterns of MS MRI presentation. This would imply that BCS is not a subtype of demyelinating disease with peculiar neuropathological and radiological features, but rather a rare manifestation of MS. Supporting this hypothesis, disease- 
Fig. 1 a Patient \#1 brain MRI. Left side a large hyperintense lesion with well-defined concentric alternating bands of myelinated and partially demyelinated white matter (T2weighted image). Right side the evolution of the lesion over 6 weeks. b Patient \#1 brain MRI. New MS-like lesions on a FLAIR scan. c Patient \#1 brain MRI. Left side new lesion on a FLAIR scan. Right side Gadolinium-enhanced T1W shows focal enhancement in the right cerebellar peduncle. d Patient \#2 brain MRI. The evolution of the lesion over 5 weeks. e Patient \#2 brain MR two-dimensional chemical shift image. Decrease of $N$ acetylaspartate peak, mild increase of choline peak and the presence of lactate peak (more evident in the core of the lesion). f Patient \#3 brain and spinal cord MRI. Left side two large gadolinium ringenhancing lesions with lamellar concentric features. Right side cervical non-enhancing MS plaques
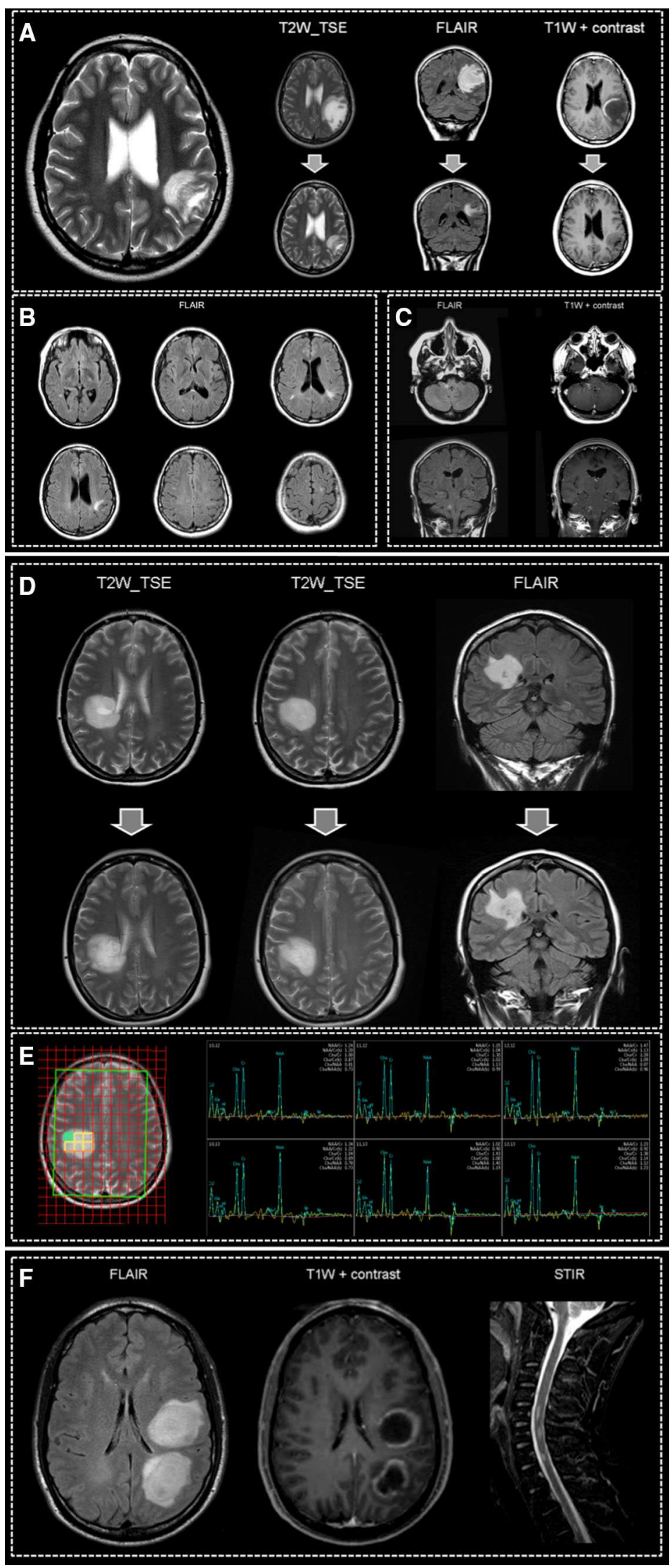
modifying therapy might be a valid treatment option in some patients, who otherwise fulfil diagnostic criteria for MS, as confirmed by our cases.

Acknowledgments This research received no specific grant from any funding agency in the public, commercial, or not-for-profit sectors.

\section{Compliance with ethical standards}

Conflict of interest Authors declare that there is no conflict of interest.

\section{References}

1. Karaarslan E, Altintas A, Senol U et al (2001) Baló's concentric sclerosis: clinical and radiologic features of five cases. Am J Neuroradiol 22(7):1362-1367

2. Capello E, Mancardi GL (2004) Marburg type and Balò's concentric sclerosis: rare and acute variants of multiple sclerosis. Neurol Sci 25:S361-S363
3. Khiat A, Lesage J, Boulanger Y (2007) Quantitative MRS study of Balò's concentric sclerosis lesions. Magn Reson Imaging 25:1112-1115

4. Ripellino P, Khonsari R, Stecco A, et al (2015) Clues on Balò's concentric sclerosis evolution from serial analysis of ADC values. Int J Neurosci (Epub ahead of print)

5. Hardy TA, Miller DH (2014) Balò's concentric sclerosis. Lancet Neurol 13:740-746

6. Mowry EM, Woo GH, Ances BM (2007) Technology insight: can neuroimaging provide insights into the role of ischemia in Baló's concentric sclerosis? Nat Clin Pract Neurol 3:341-348

7. Lucchinetti CF, Gavrilova RH, Metz I et al (2008) Clinical and radiographic spectrum of pathologically confirmed tumefactive multiple sclerosis. Brain 131(7):1759-1775

8. Hardy TA, Beadnall HN, Sutton IJ et al (2015) Baló's concentric sclerosis and tumefactive demyelination: a shared immunopathogenesis? J Neurol Sci 348(1-2):279-281

9. Barnett MH, Prineas JW (2004) Relapsing and remitting multiple sclerosis: pathology of the newly forming lesion. Ann Neurol 55:458-468

10. Kastrup O, Stude P, Limmroth V (2002) Balo's concentric sclerosis. Evolution of active demyelination demonstrated by serial contrast-enhanced MRI. J Neurol 249:811-814 